\title{
An animal experiment study on the application of indocyanine green angiography in the harvest of multi-angiosome perforator flap
}

\author{
Chaojie Wanyan", Zhongming Wu", Fengrui Zhang, Huan Li, Zihui Yang, Jun Wang, Xuejiao Han, \\ Xiangming Yang, Delin Lei, Xinjie Yang, Jianhua Wei
}

State Key Laboratory of Military Stomatology, National Clinical Research Center for Oral Diseases, Shaanxi Clinical Research Center for Oral Diseases, Department of Oral and Maxillofacial Surgery, School of Stomatology, The Fourth Military Medical University, Xi'an, China

Contributions: (I) Conception and design: C Wanyan; (II) Administrative support: J Wei; (III) Provision of study materials or patients: Z Wu; (IV) Collection and assembly of data: F Zhang; (V) Data analysis and interpretation: X Yang; (VI) Manuscript writing: All authors; (VII) Final approval of manuscript: All authors.

\#These authors contributed equally to this work.

Correspondence to: Jianhua Wei. State Key Laboratory of Military Stomatology, National Clinical Research Center for Oral Diseases, Shaanxi Clinical Research Center for Oral Diseases, Department of Oral and Maxillofacial Surgery, School of Stomatology, The Fourth Military Medical University, Xi'an 710032, China. Email: weiyoyo@fmmu.edu.cn.

Background: This study sought to explore the application value of indocyanine green angiography (ICGA) in the harvest of multi-angiosome perforator flap and the effect of low molecular weight heparin (LMWH) on the survival of postoperative flap.

Methods: Twenty-four SD male rats were selected to construct a three-angiosome perforator flap model with the unilateral iliolumbar artery perforator. They were randomly divided into two groups: the control group was injected with indocyanine green (ICG) into the femoral vein during the operation, and the fluorescence signal was collected and quantitatively analyzed using Real-Time Image Guided System to determine the intraoperative fluorescence imaging length. The experimental group was injected subcutaneously with $\mathrm{LMWH}(400 \mathrm{U} / \mathrm{kg})$ after $0.5 \mathrm{~h}$ postoperatively, and the control group was injected with the same amount of normal saline. The injection was repeated at the same time each day from 0 to 7 days postoperatively. After the flap was sutured in situ, ICGA was performed at 0, 1, 3, 5, and 7 days postoperatively to observe the vascular structure of the two groups of flaps. The flap survival length of the control group was counted at 7 days postoperatively, and the correlation between the intraoperative fluorescence imaging length and the survival length at 7 days postoperatively was calculated. The proportion of distal necrosis of the flaps between the two groups was compared at 7 days postoperatively.

Results: The average length of intraoperative fluorescence imaging in the control group was $6.29 \pm 0.50 \mathrm{~cm}$, and the survival length of the flap at 7 days postoperatively was $8.24 \pm 0.52 \mathrm{~cm}$. The actual survival length was higher than the intraoperative fluorescence imaging length, with a ratio of $1.31 \pm 0.08$. The difference was statistically significant $(\mathrm{P}<0.05)$. At 7 days postoperatively, the flap necrosis ratio of experimental group and control group were $10.92 \% \pm 1.30 \%$ and $19.11 \% \pm 1.19 \%$, and the flap necrosis ratio of experimental group was lower than that of control group $(\mathrm{P}<0.001)$.

Conclusions: ICGA can locate the position of perforator, and can be used to predict and observe the length of distal survival of multi-angiosome perforator flap postoperatively. LMWH can promote the distal survival of flap and reduce flap necrosis.

Keywords: Indocyanine green angiography; angiosome; perforator flap; limit length; low molecular weight heparin

Submitted Dec 07, 2021. Accepted for publication Feb 14, 2022.

doi: $10.21037 /$ atm-22-220

View this article at: https://dx.doi.org/10.21037/atm-22-220 


\section{Introduction}

The perforator flap is defined as an axial flap with small skin vessels (perforating muscles or intermuscular septa $\geq 0.5 \mathrm{~mm}$ ) (1). Since Koshima et al. (2) first described the perforator flap in 1989 , its clinical application has become increasingly widespread due to its flexible design, minimal invasiveness, and the reduction of donor site complications (3). However, there are large variations in the caliber and location of the perforator vessels, and the improper positioning of the perforator fulcrum and intraoperative assessments of the distal blood perfusion of the flap often cause complications, including flap harvest failure, the distal ischemia of long flaps, infection, wound dehiscence, and necrosis (4). The objective detection of flap perfusion in real time is key to flap preparation (5). At present, there are various techniques for locating perforator and detecting flap perfusion, but there are no objective unified standards (6).

Over the past few decades, near-infrared fluorescent imaging for medical diagnosis has been employed extensively in the medical area. As a contrast agent, indocyanine green (ICG) was initially used in cardiology, ophthalmology and hepatology, but now it is most widely used in tumor recognition, organ scintigraphy, lymphatic tracing and blood flow monitoring (7). Furthermore, ICG-based applications have gained increasing relevance in reconstructive surgery over the years, including preoperative flap design, intraoperative tissue perfusion evaluation and postoperative flap monitoring (8). indocyanine green angiography (ICGA) has been shown to improve the success rate of flap transplantation and reduce flap necrosis $(8,9)$. It was a series of cases in the report showing the usage of ICGA in flap harvest, which was the authors' experience in a kind of descriptive manner (10). In the present study, we did quantitative study on the multiperforator flap harvest by means of ICGA in animal model, and we also did intervention study by employing low molecular weight heparin (LMWH). We believe our results are helpful for larger perforator flap harvest and postoperative free flap management.

Long free flap is needed in some clinical scenario. Although several workhorse flaps may meet the need, the donor site damage will be remarkable. Multi-angiosome perforator flap fills the gap with balance between the donor and recipient site. Due to high variability in perforator size and course, skilled microvascular manoeuvre remains a major challenge for surgeons. In most cases, only one perforator is anastomosed because limited recipient vessels and clinical need for flexible manipulation in flap inset. As distal end necrosis may happen in multi-perforator flap, how to design and verify the vitality of distal end of flap is badly needed in clinical practice (11). Multi-angiosome flap usually supply multiple angiosomes by a single vascular pedicle, and the distal end of the flap is prone to ischemic necrosis due to hypoperfusion. There are a variety of imaging methods to assess the perfusion range of perforators before or during surgery to harvest flaps suitable for covering the wound; however, flap transplant-related complications may still occur after surgery, and timely intervention is essential.

Delayed technique is an effective method for increasing the area of the flap and opening the subcutaneous microcirculation of the flap (12). Surgical delays are generally 3-4 weeks in length, result in multiple surgeries, and have related problems, such as long hospital stays and increased clinical costs. The issue of whether the ultimate length of the distal survival of perforator flaps can be improved by drugs is a clinical concern (13). LMWH has anticoagulant, antithrombotic and anti-inflammatory effects with few adverse reactions. It is mainly used for the prevention of VTE in hypercoagulable patients. In some studies, LMWH was used in perforator flap surgery for anticoagulation postoperatively to reduce flap compromise. At present, it has become a routine anticoagulant drug after flap surgery in many centers, but if it can increase limit length of multi-angiosome perforator flap is to be clarified (14). However, to date, no study has been conducted on the effect of multi-angiosome flap survival. Using a new generation of fluorescence imaging equipment, we designed this experiment to examine whether ICGA can be used to predict and observe the limit range of distal flap survival after surgery, and whether LMWH promotes the survival of cross-regional flaps after surgery to provide an experimental basis for the reasonable harvest of flaps and the reduction of flap necrosis.

We present the following article in accordance with the ARRIVE reporting checklist (available at https://atm. amegroups.com/article/view/10.21037/atm-22-220/rc).

\section{Methods}

\section{Materials}

Experimental animals: 24 healthy adult male Sprague Dawley rats, Specific Pathogen Free (SPF), grade aged 6-8 weeks, weighing $300 \pm 10 \mathrm{~g}$, were provided by the Animal Experimental Center of The Fourth Military 
Medical University (license no. SYXK[Shan]2019-001). The rats were kept under a 12 -h day-night cycle at $25^{\circ} \mathrm{C}$ and a humidity of $50 \%$, and were allowed free access to food and water. Experiments were performed under a project license (No. 20200637) granted by ethics board of The Fourth Military Medical University, in compliance with national guidelines for the care and use of animals.

The main reagents and equipment were as follows: indocyanine green (ICG) for injection (25 mg/vial, Dandong Yichuang Pharmaceutical Co., Ltd.); the surgical fluorescence imaging system FLI-10B series (Real-IGS FLI-10B; Nanjing Noyuan Medical Instrument Co., Ltd., Nanjing, China); and a LMWH calcium injection (2,500 U/vial, Hefei Zhaoke Pharmaceutical Co., Ltd.).

\section{Experimental grouping and methods}

Twenty-four rats were randomly numbered 1 to 24 and equally divided into LMWH intervention group (the experimental group) and the saline group (the control group); 12 rats per group.

\section{The multi-angiosome flap model was established}

The rats were fasted for 1 day before the operation. After being anesthetized via an intraperitoneal injection of $5 \%$ pentobarbital sodium $(50 \mathrm{mg} / \mathrm{kg})$, each rat was fixed to the operating table in the prone position and depilated on the right side of the back. The probe of FLI-10B was moved to a focus distance of $40 \mathrm{~cm}$ to the right back, $0.05 \mathrm{~mL}$ of ICG solution at a concentration of $2.5 \mathrm{mg} / \mathrm{mL}$ was administered via the tail vein, and FLI-10B detection was started and videotaped during the injection. In the process of dynamic imaging, the earliest or brightest point was defined as the location of the perforator vessel. The iliolumbar perforator fulcrum was marked in the area of the iliolumbar perforator of the flap. At this point, the flap was extended $1 \mathrm{~cm}$ to the tail as the rear boundary, $11 \mathrm{~cm}$ to the head as the front boundary, $2 \mathrm{~cm}$ to the right side of the back midline as the inner boundary of the flap, and $1 \mathrm{~cm}$ to the back of the proximal axillary line as the outside. The size of the rectangular flap was $3 \mathrm{~cm} \times 12 \mathrm{~cm}$. The flap cut profile was marked.

After disinfection with 5\% iodophor, the medial side of the flap was first incised, the flap was separated from the inside to the outside, the fascia was carefully incised and bluntly separated, attention was paid to preserving the iliolumbar perforator, and the other boundaries of the flap were then incised to free the iliolumbar perforator, intercostal perforator, and thoracodorsal perforator. Next, the flap was lifted, and ICGA was re-performed. After the metabolism of ICG in vivo, the intercostal perforator, thoracodorsal perforator, and all tissues around the flap were divided to prepare a 3-vessel body area ultra-long perforator flap with only the iliolumbar perforator.

In the control group, after successful flap preparation by an intraoperative ICGA examination, FLI-10B video recording dynamic angiography was initiated. The ICG solution was injected, and images were taken for the quantitative analysis when fluorescence extended from the iliolumbar puncture fulcrum distally to the flap to reach stability (approximately $30 \mathrm{~s}$ after injection). In the previous experiment, we found that the fluorescence intensity base point of the surrounding background, and the area without ICG perfusion in the flap was 20-30 arbitrary units (AU), with a "dark area", indicating no blood flow signal, and the fluorescence intensity base point value of the area with ICG perfusion in the flap was 30-255 AU, with a "bright area", indicating blood flow signal. The captured images were imported into ImageJ software for analysis, and the fluorescence intensity threshold was set to $30 \mathrm{AU}$, which was used for the region segmentation, and the fluorescence imaging area and non-imaging area were delineated to represent the intraoperative flap blood perfusion area and no blood perfusion area, respectively, and the fluorescence boundary line was generated. The short axis of the flap where the perforator fulcrum was located was depicted by a black line segment, and this line was divided into 6 equal parts; the 6 equal points made 5 red line segments intersecting the fluorescence boundary line in the direction of the long axis of the parallel flap, and the average length of these 5 -line segments was recorded as the intraoperative fluorescence imaging length (see Figure 1).

\section{Administration time and method experimental group} LMWH calcium (400 U/kg) was subcutaneously injected around the umbilicus beginning at $0.5 \mathrm{~h}$ after the operation, and then once every $12 \mathrm{~h}$, and continuously injected at the same time every day from postoperative days 0 to 7 . The control group was given the same amount of saline at the same time points. In both groups, the flaps were intermittently sutured in situ with 5-0 nylon sutures. At the end of surgery, antibiotic ointment was applied, and each rat was fed in a single cage in the same housing environment. Each rat received 100,000 U/day of penicillin subcutaneously 0-3 days after surgery. All the surgical designs and procedures were performed by the same person. 


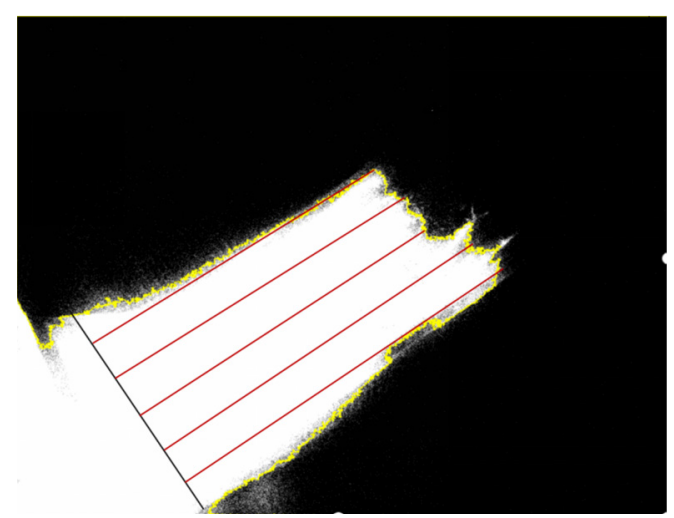

Figure 1 Method for measuring intraoperative fluorescence imaging length.

\section{Postoperative flap observation and measurement indicators \\ Gross observation of the flap and comparison of the necrotic area between the 2 groups}

At postoperative days 1-7, the flap survival status, including the flap shape, color, tissue elasticity, and texture, was observed macroscopically in both groups. At postoperative day 7 , the flap survival of rats in each group was photographed and analyzed with ImageJ software. The area of rat flap necrosis was measured, and the flap necrosis ratio was calculated according to the flap necrosis criteria (i.e., black color, poor tissue retraction, elasticity, hard texture, and no bleeding from the cut tissue). Flap necrosis ratio $=$ necrotic area/total area $\times 100 \%$.

\section{Correlation analysis between intraoperative fluorescence imaging length and flap survival length on postoperative day 7}

At postoperative day 7, the flap survival length of the rats in the control group was calculated, and the correlation analysis was performed between the intraoperative fluorescence imaging length and the flap survival length at postoperative day 7 .

\section{Postoperative flap blood supply analysis}

The blood flow changes for the flaps in the 2 groups were recorded within 7 days of the operation using the color scale mode, and the color scale images were analyzed to examine the relative perfusion of blood flow using Image $\mathbf{J}$ software (a circular region of interest with a radius of $5 \mathrm{~mm}$ was set at the center of the iliolumbar perforator fulcrum to obtain the mean fluorescence intensity value of this region, which was multiplied by 0.8 as the threshold segmentation point to convert the color scale images into black-and-white images; white indicated that the fluorescence intensity was greater than this threshold, which was a relatively rich perfusion region, while black indicated that the fluorescence intensity was less than this threshold, which was a relatively not rich perfusion region. The changes of blood supply in the flaps over time were also visually observed. A quantitative analysis of relatively rich and not rich perfusion was conducted using the color scale mode images; the origin represented the location of iliolumbar perforator fulcrum; the $\mathrm{X}$-axis indicated that the long axis of the flap(from iliolumbar perforator fulcrum to the distal end of the flap), and the position of each point on the $\mathrm{X}$-axis is expressed in pixels, representing the distance from each point of the axis to the fulcrum; the Y-axis represents the fluorescence intensity of each point on the $\mathrm{X}$-axis; the intersection point between the longitudinal line and the $\mathrm{X}$-axis represented the relatively rich and not rich perfusion demarcation line, and the dynamic changes of the demarcation line over time were observed.

\section{Statistical analysis}

All the data are expressed as mean \pm standard deviation $(\bar{x} \pm s)$, and the paired $t$-test and 2-sample $t$-test were performed with SPSS 21.0 software. Linear fitting was performed on the data with Origin2021 software. A $\mathrm{P}$ value $<0.05$ was considered statistically significant.

\section{Results}

\section{Establishment of the 3-angiosome ultra-long flap model}

ICGA can accurately locate the iliolumbar perforator fulcrum and thoracodorsal perforator fulcrum on the back of rats, but the intercostal perforator fulcrum cannot be clearly displayed due to the strong fluorescence interference of the heart, and the intercostal perforators being adjacent to the heart. The flap harvesting range was determined according to the location of the iliolumbar perforator fulcrum. ICGA after flap lifting clearly showed that the blood in the flap was supplied by the iliolumbar perforators, intercostal perforators, and thoracodorsal perforators. All the tissues except the iliolumbar perforators were divided, and the 3 -angiosome ultra-long perforator flap was successfully prepared (see Figure 2).

\section{Intraoperative ICGA results}

In the control group, ICGA was performed during the operation. The first imaging showed the iliolumbar angiosome. After this area became darker, the intercostal 

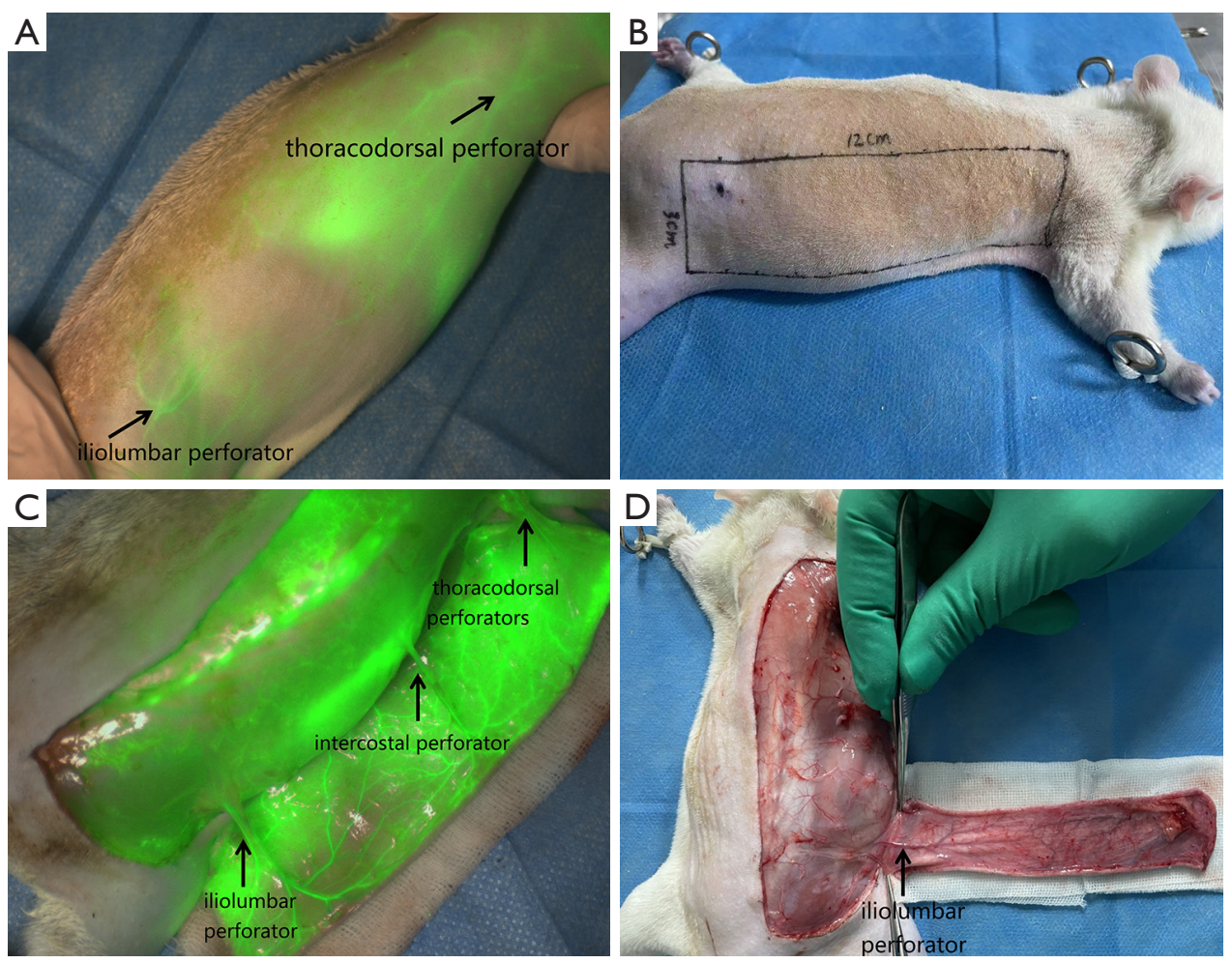

Figure 2 Flap design and the harvest of the 3-angiosome perforator flap. (A) The location of the perforator; (B) flap design; (C) three perforators upon flap elevation (arrow); (D) flap harvest based on the iliolumbar perforator (arrow).

angiosome began to be imaged, but the brightness was slightly darker; generally, the thoracodorsal angiosome was only weakly imaged or not imaged. The key images were taken when fluorescence expansion reached stability. The whole flap was only supplied by the iliolumbar perforator, and the fluorescence intensity gradually decreased from the flap to the distal end, with the flap presenting a "bright area" and a "dark area" at the distal end (see Figure 3).

\section{Observation and measurement analysis of the postoperative flaps}

\section{Gross morphological observations}

The rats in both groups survived until the completion of the experiment. After flap harvest and suturing in situ, some degree of cyanosis appeared at the distal end of the flap. At postoperative day 1 , the flaps showed different degrees of swelling, and were dark purple at the distal end. At postoperative day 3, the dark purple area at the distal end expanded, the color deepened to dark brown, and the demarcation between the necrosis and survival areas was unclear. At postoperative day 5, the distal end of the flap in the control group had a black scab shell, the texture hardened, showing dry necrosis, and the necrosis area was stable. At postoperative day 7 , the flap performance in the control group was consistent with that at postoperative day 5 , and the distal necrotic part of the flap in the experimental group tended to fuse, the boundary line was clear, the flap survival quality was good, and the degree of contracture was mild (see Figure 4). The flap necrosis ratio in the experimental group was $10.92 \% \pm 1.30 \%$ at postoperative day 7, which was significantly lower than that of $19.11 \% \pm 1.19 \%$ in the control group $(\mathrm{t}=17.261 ; \mathrm{P}<0.001$; see Figure 5 and Table 1).

\section{Correlation analysis between fluorescence imaging length and flap survival length at postoperative day 7} After the statistical analysis, the actual survival length of the flap was greater than the intraoperative fluorescence imaging length $(\mathrm{P}<0.05)$, and the difference in length between the two was statistically significant, with a length ratio of $1.31 \pm 0.08$ (see Table 2). The correlation coefficient of linear fitting analysis was $r=0.7124$, indicating that the length between the two has a strong correlation $(\mathrm{P}<0.05)$ (see Figure 6). 

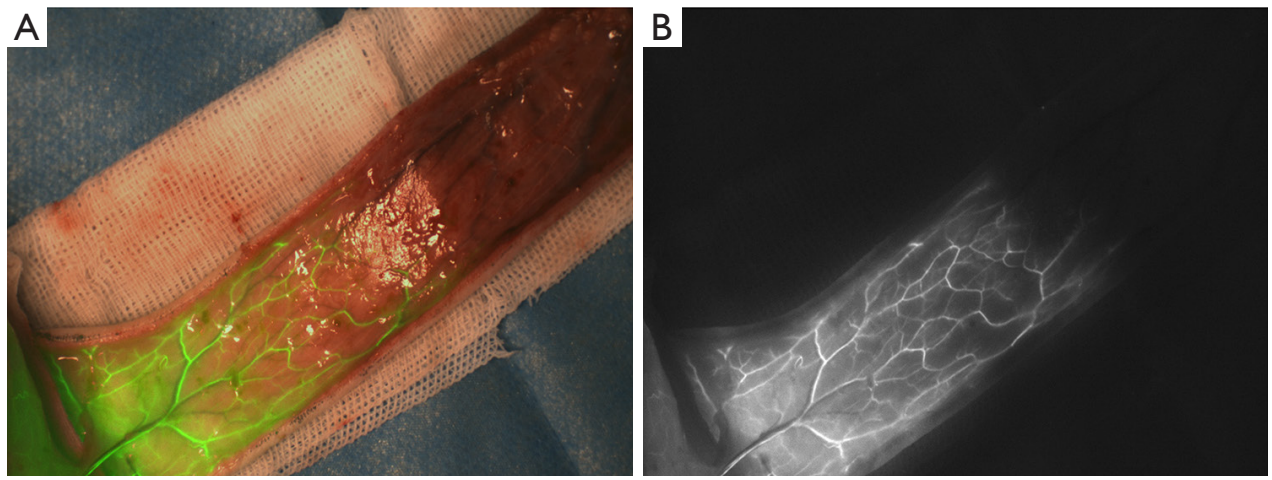

Figure 3 Intraoperative flap fluorescence imaging. (A) Merged mode; (B) fluorescence mode.
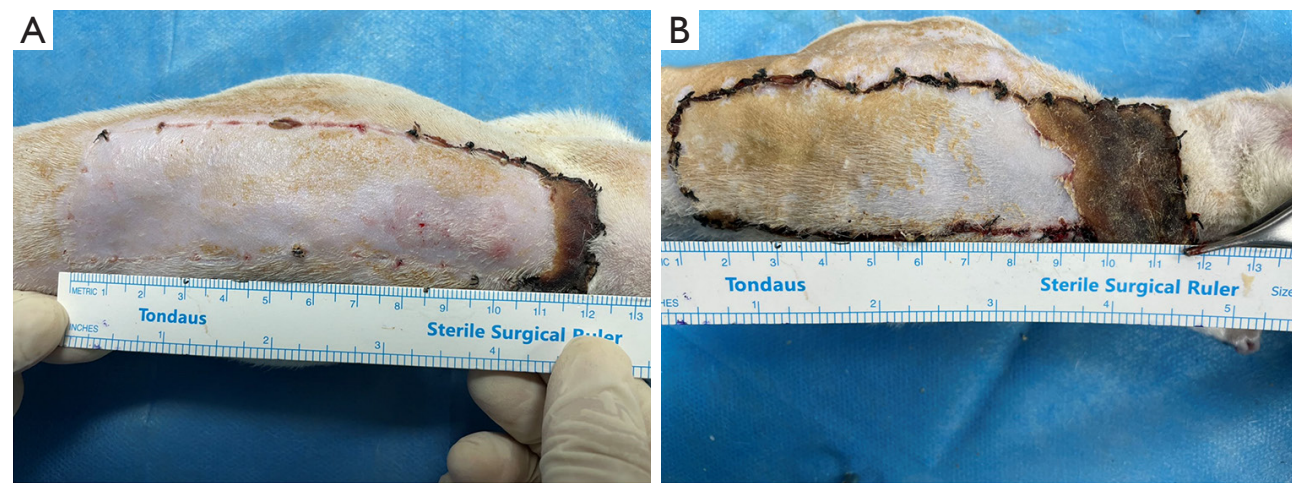

Figure 4 Gross observation of flaps at postoperative day 7. (A) Gross observation of a flap in the experimental group; (B) gross observation of a flap in the control group.

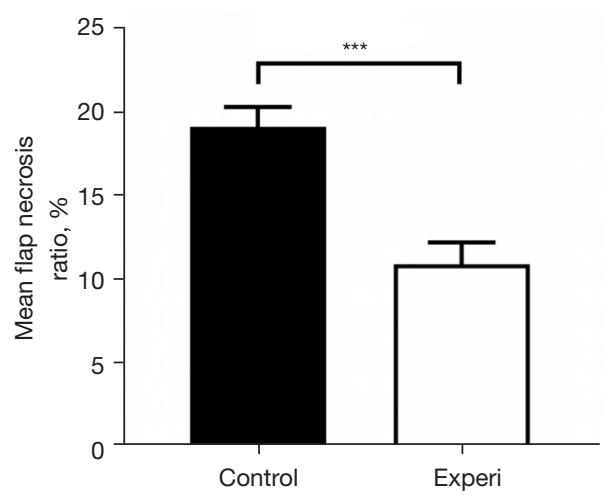

Figure 5 Comparison of flap necrosis between the 2 groups. ${ }^{* * *} \mathrm{P}<0.001$.

\section{Postoperative flap blood supply analysis}

After the formation of a flap pedicled with the iliolumbar perforator, the flap blood was supplied only by the iliolumbar perforator. Color scale images suggested that at postoperative days 0 and 1 , the flap fluorescence intensity was weak in both groups. The quantitative analysis showed that the fluorescence of the angiosome of the iliolumbar perforator was relatively weak, and the middle and distal ends of the flap were relatively weak. At postoperative day 3 , the blood supply of the flap was significantly increased, the fluorescence of the flap and the middle segment was significantly enhanced, the fluorescence development area was gradually increased, the intersection moved distally, and the distal blood supply was improved. The boundary between the rich and not rich perfusion areas was not clear. Compared to the control group, the flaps of the experimental group were significantly improved. At postoperative days 5 and 7, the flap blood flow of the 2 groups continued to improve, the fluorescence development area continued to expand, the "bright" and "dark" areas remained stable, and the boundary was obvious. Additionally, the intersection point continued to move distally, and the curve decreased abruptly at the same position at the distal end of the $\mathrm{X}$ axis, indicating that the flap blood 
Table 1 Comparison of the flap necrosis area and necrosis ratio at postoperative day 7 between the 2 groups $\left(\bar{x}_{ \pm} \mathrm{s}\right)$

\begin{tabular}{lcccc}
\hline Group & No. of subjects & Mean flap necrosis area $\left(\mathrm{mm}^{2}\right)$ & Mean total flap area $\left(\mathrm{mm}^{2}\right)$ & Mean flap necrosis ratio $(\%)$ \\
\hline Control & 12 & $624.33 \pm 24.95$ & $3277.48 \pm 217.02$ & $19.11 \pm 1.19$ \\
Experimental & 12 & $364.13 \pm 45.87$ & $3335.84 \pm 124.57$ & $10.92 \pm 1.30$ \\
\hline
\end{tabular}

Table 2 Analysis of the survival length of flaps at the 7th day and the length of intraoperative fluorescence imaging

\begin{tabular}{lcc}
\hline Animal serial number & Survival length $(\mathrm{cm})$ & Intraoperative fluorescence imaging length $(\mathrm{cm})$ \\
\hline 1 & 8.10 & 5.68 \\
2 & 8.27 & 5.84 \\
3 & 8.36 & 6.00 \\
4 & 8.84 & 6.91 \\
5 & 7.09 & 6.08 \\
6 & 8.38 & 6.18 \\
7 & 8.60 & 6.56 \\
8 & 7.26 & 5.49 \\
9 & 8.20 & 6.27 \\
10 & 8.74 & 7.11 \\
11 & 8.39 & 6.33 \\
12 & 8.66 & 7.01 \\
All & $8.24 \pm 0.52$ & $6.29 \pm 0.50$ \\
\hline
\end{tabular}

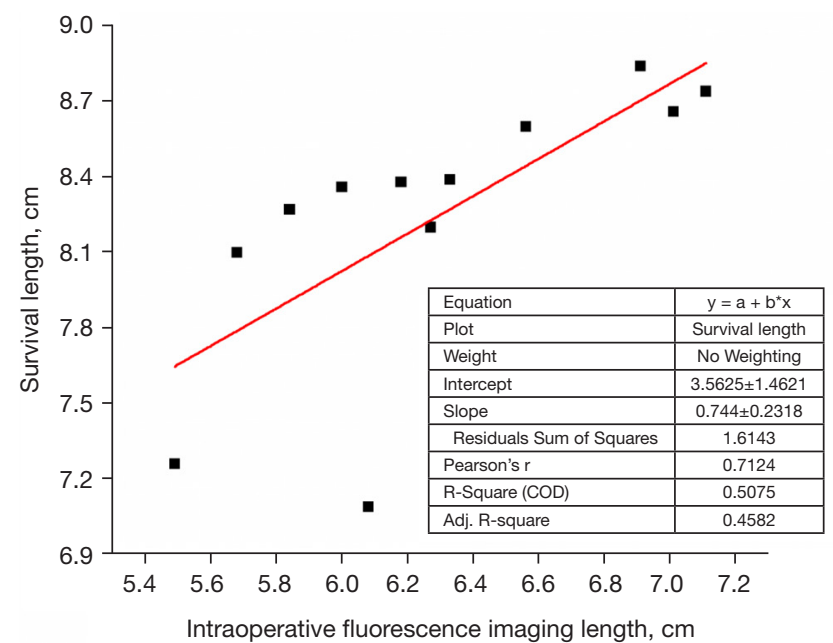

Figure 6 Linear fitting analysis of the survival length of the flap at postoperative day 7 and the length of intraoperative fluorescence imaging. flow of both groups had been stable at postoperative day 5 . The flap eventually formed a blood supply system centered on the iliolumbar perforator (see Figure 7).

\section{Discussion}

In the past 30 years, with the development of microsurgical techniques, the success rate of various types of perforator flaps in the field of reconstructive surgery has increased, but there is still a failure rate of $2-4 \%$ (15). The occurrence of flap adverse events is closely related to the large anatomical variation of the perforator vessels, and an inability to effectively assess donor site tissue perfusion (16). In fact, large-scale tissue defects often occur in clinical practice. The traditional perforator flap directly supplies a small anatomical area, but cross zone flap is expected to repair a large range of defects (17). In 1987, Taylor (18) proposed the "angiosome" theory that states that the donor site of 
A
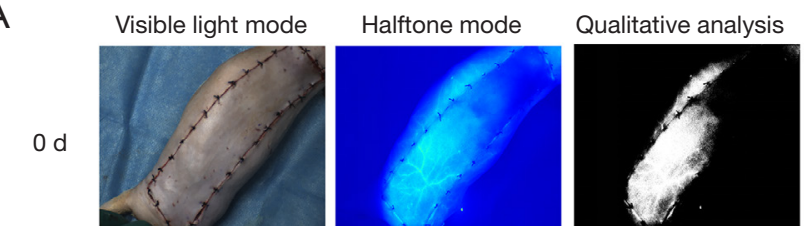

Quantitative analysis

d
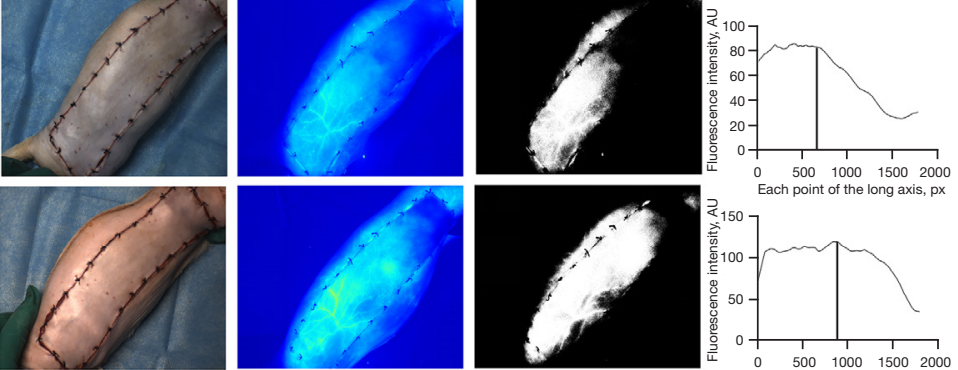

$3 d$
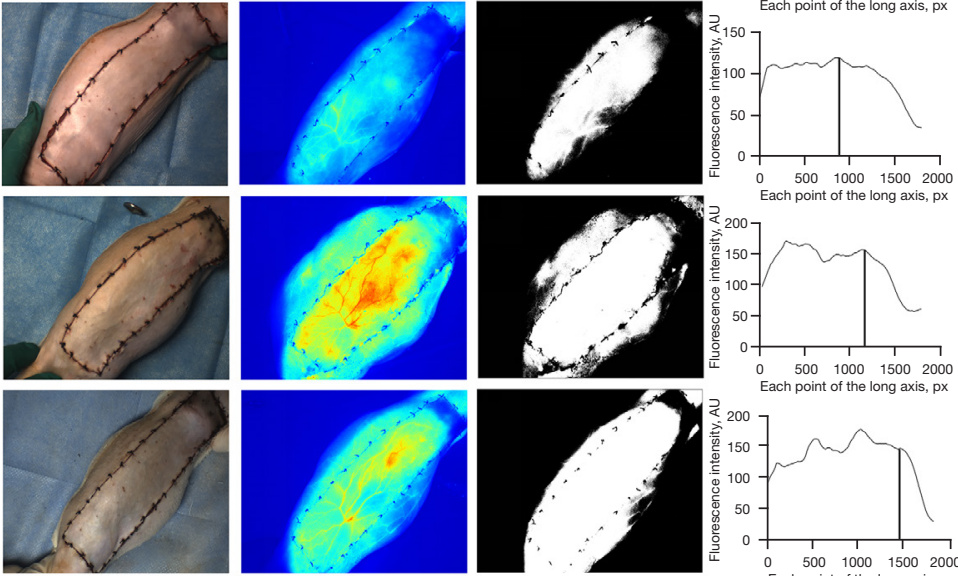

$7 \mathrm{~d}$
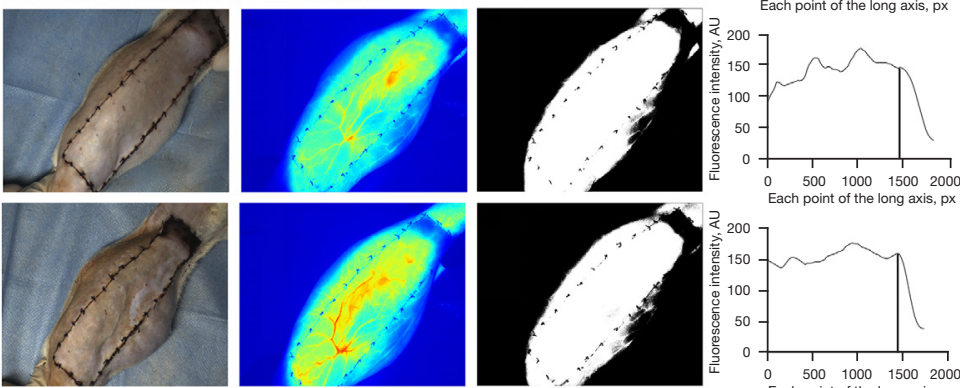

B

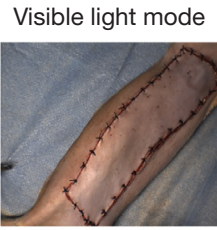

Halftone mode

Qualitative analysis

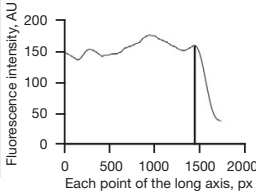

$0 d$
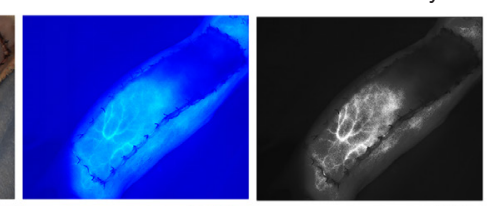

Quantitative analysis
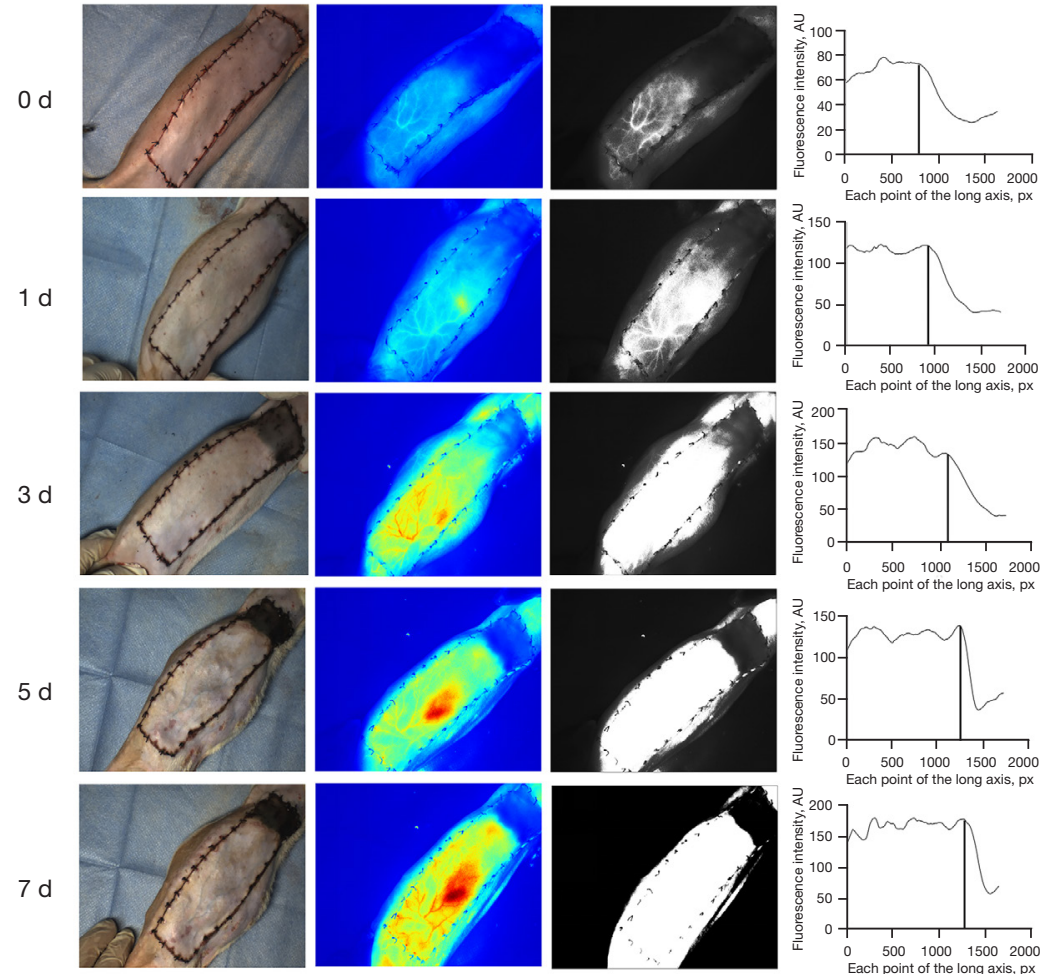

Figure 7 Analysis of blood supply of the flap postoperatively. (A) Analysis of blood circulation after flap surgery in the experimental group; (B) analysis of blood circulation after flap surgery in the control group. 
each well-known perforator vessel not only includes the skin and subcutaneous tissue within its distribution range, but also the 3-dimensional structure of the corresponding muscles, tendons, bones, and other tissues in the same area. Cross-regional flaps contain 2 or more vascular body areas, and the blood vessel network between the angiosomes that gradually decrease in diameter and are mutually anastomosed is called the "choke vessels". Vessels in the choke zone play a bridging role in the survival of cross-area flaps, and studies have shown that when a flap pedicled with only one perforator spans 3 angiosomes, necrosis of the flap often occurs in the choke anastomosis area between the kinetic and potential donor sites (19).

The question of how the multi-angiosome flap can be evaluated to determine the cut-off length and avoid distal necrosis is currently the focus of research (20). At present, the tissue perfusion assessment tools commonly used in clinical practice include the laser Doppler flow (LDF) monitor, infrared thermography (IRT), and spectral analysis (21). The LDF monitor allows the flap microcirculatory status to be determined intraoperatively, but the results may underestimate the flap viability (22). IRT reflects range of flap perforating blood supply and responds rapidly to changes in blood flow, but is susceptible to external factors, such as room temperature and humidity (23). A spectral analysis indirectly assesses tissue by monitoring tissue conditions, but the operation time is long and the data are susceptible to noise (21).

ICG is one of the most commonly used near-infrared fluorescent dyes in medicine, which is widely used in the diagnosis and treatment of many cancers (24). It binds to plasma proteins that confine most of the bolus to the intravascular space until hepatic uptake and excretion into bile with no harm to surrounding tissues. The dye is cleared from the system exponentially in the first 10 to 20 min after application, with a half-life of generally 3 to 4 min depending on the vascularization of the organ of interest (25). Therefore, multiple injections can be repeated in a short period of time. In contrast, other near-infrared fluorescent dyes such as methylene blue and sodium fluorescein have a clearance time of more than $12 \mathrm{~h}$, and cannot be used multiple times in a single procedure, and are less accurate and sensitive than ICG (26). ICG is virtually nontoxic, provided the patient have no iodide allergy (27). The well-tolerated interactions and reactions of ICG in the body are useful in medical applications because they pose low risk to the patient. Unlike other techniques, ICGA provides intuitive, dynamic, and real-time flap blood flow images, and the corresponding perforator perfusion range. ICGA is applied preoperatively or intraoperatively to assist in flap harvest (28). At present, the most widely used ICGA is the SPY system (NOVADAQ Company, Canada), which has its own SPY-Q software, and provides the following 2 perfusion assessment parameters: absolute perfusion value and relative perfusion value. The absolute value refers to the absolute fluorescence intensity of the study area, and the relative value refers to the fluorescence ratio of the study area to the reference area using the maximum perfusion area as the reference area. Moyer (29) observed the blood supply of 118 mastectomy donor site flaps using ICGA, and found that $90 \%$ of the tissues with relative values $\leq 25 \%$ were perfused, and $90 \%$ were non-viable, while $98 \%$ of the tissues with relative values $\geq 45 \%$ were viable. Phillips et al. (30) investigated the necrosis threshold using the absolute value as an evaluation parameter for chest flaps in 51 cases of breast reconstruction, and found that flaps had a high risk of necrosis when the absolute value was $<3.7 \mathrm{AU}$, and had viable tissue when the absolute value was $>8.0 \mathrm{AU}$. When the fluorescence value is 3.7-8.0 AU, the flap needs to be evaluated combined with clinical experience. The absolute value reflects the true fluorescence intensity in the study area, but is susceptible to temperature, ICG concentration, individual patient condition, etc. (31). Due to the strong subjectivity of the reference area selection, there are great variations between different types of flaps and individuals, and there is no uniform standard for the obtained relative indicators in different individuals. The sensitivity of each fluorescence imaging device also differs, and no clear consensus has been reached as to the hemoperfusion cut-off value for predicting flap outcomes.

Compared with the traditional SPY system, the FLI10B model has a higher sensitivity for ICG detection concentration of 1-10 nM, a large area light source of $100 \mathrm{~cm}^{2}$, laser uniformity $>8.0 \%$, and can completely image the surgical field. Additionally, the built-in software is further optimized, and it is exclusively equipped with two quantitative analysis systems of base point value and spectral value, which can be quantitatively analyzed during surgery. It has a color scale mode that can be used for blood supply observation. In this experiment, FLI-10B was used to locate the perforation point first, and then the 3-angiosome perforator flap model proposed by Yang et al. (32) was established. We set the fluorescence intensity threshold to $30 \mathrm{AU}$, such that a fluorescence value $>30 \mathrm{AU}$ indicated blood perfusion. Compared to the previous application of relative perfusion indicators, this method 
avoids subjective selection error by setting a constant threshold without selecting a reference area, which should provide a uniform evaluation criterion for different types of flaps and different individuals. Additionally, unlike absolute perfusion indicators, this method does not determine flap survival and necrosis only by setting a cut-off value, but objectively and quantitatively analyzes the correlation between intraoperative fluorescence imaging length and postoperative flap survival length, reduces the effects of ICG concentration, temperature, and surgical stimulation, and thus increases the objectivity and accuracy of the measurement results. Our results suggests that the flap harvest limit length is greater than the intraoperative imaging length, and the safe range for predicting flap survival according to angiography maximizes the repair of the defect while avoiding distal necrosis. As for the ability to determine the exact length of flap harvest, further validation is needed.

The effective blood supply area of the cross-regional flap can be initially confirmed by ICGA, but sometimes there is still partial necrosis at the distal end after flap harvesting and transfer. Surgical delay promotes telangiectasia and proliferation in the choke area, improves microcirculation at the distal end of the flap, and thus improves flap survival (33-35). Chemical delay avoids the pain associated with multiple surgeries, promotes vascular proliferation through intermittent hypoxia on the flap, expands the vascular caliber, and increases the blood supply area. At present, the drugs that promote the survival of skin flaps include vasodilators nitroglycerin, epinephrine, vascular endothelial growth factor (VEGF) (13). Inadequate blood supply and ischemiareperfusion injury are major factors of flap necrosis (36). In clinical practice in recent years, LMWH has become a commonly used anticoagulant drug. It not only has antivenous congestion and prostrate response effects, but can also be used for the treatment of ischemia-reperfusion injury (37). However, it has not been reported whether it can inhibit the ischemia-reperfusion injury of the cross-regional flap and increase the blood supply of the distal flap. We were pleasantly surprised to find that the application of $\mathrm{LMWH}$ significantly increased the survival limit length of the flap, and no local bleeding occurred after surgery. Thus, LMWH appears to promote the distal survival of cross-boundary flaps with some delayed effect, and the dose used is also within the safe range. However, the specific mechanism and exact effect of delay need to be studied further.

Conventional angiographic techniques only show the static vascular state, but ICGA provides an image of the blood flow passage under the physiological state, which reflects the whole process of blood flow change and vascular remodeling (38). Postoperative monitoring revealed that blood flow was weak at days 0 and 1 and significantly increased at day 3. Consistent with the findings of Dhar et al. (35), we found that the choke vessels are temporarily in a contractile state for $48 \mathrm{~h}$ after surgery, and the choke vessels rapidly expand and the vascularity is reconstructed along the direction of the flap at 48-72 h after surgery. Additionally, after adding $\mathrm{LMWH}$, the distal blood flow of the flap of rats in the experimental group increased more significantly on the 3rd day than that of the control group. In the control group, the survival area and necrotic area also reached a stable state, and the "dark area" was consistent with the necrotic area of the flap in the future. In the experimental group, 5 days after surgery, the extent of the "bright area" and "dark area" in the flap fluorescein angiography was stable, while the boundary of the flap necrosis area was unclear, which suggests that we can predict the risk of potential necrosis by ICGA and conduct an intervention for the potential necrosis area 5 days after surgery. Thus, hemodynamic findings observed by ICGA are more reliable than macroscopic observations at predicting the extent of flap survival.

This study had some limitations. First, there is no uniform standard as to when ICG should be used and the concentration of ICG that should be used. Many factors affect ICGA, such as temperature, ICG concentration, light, and each patient's state, but these factors have not been closely examined. The optimal dose, injection time, and specific mechanism by which LMWH promotes flap survival require further investigation. Second, the physiological structure of rats is quite different to that of humans, but the porcine flap is similar to that of humans in terms of vascular anatomy, harvest, tissue structure, and the flap healing process. Pigs should be used in future research to gradually realize the transformation to clinical practice.

\section{Conclusions}

In summary, we found preliminary evidence that ICGA can be used in the harvest of multi-angiosome perforator to localize flap perforator vessels, and predict and observe the limit range of postoperative distal flap survival, which can be further enhanced by a postoperative LMWH intervention. As animals differ physiologically from humans, we will next investigate the role of ICGA in the harvest of multiangiosome perforator flaps in vivo with the help of Real-IGS FLI-10B, which has been clinically validated. The specific mechanism by which $\mathrm{LMWH}$ promotes flap survival was 
also studied. This animal experiment provides a more exact experimental basis for subsequent clinical studies.

\section{Acknowledgments}

Funding: This work was supported by grants from National Natural Science Foundation of China (81973114) and Shaanxi Key Research and Development Program (2018SF163). This research was also funded by National "Thirteenth Five-Year" Key R\&D Program Digital Medical Equipment R\&D Special Funding Project (2016YFC0104100).

\section{Footnote}

Reporting Checklist: The authors have completed the ARRIVE reporting checklist. Available at https://atm. amegroups.com/article/view/10.21037/atm-22-220/rc

Data Sharing Statement: Available at https://atm.amegroups. com/article/view/10.21037/atm-22-220/dss

Conflicts of Interest: All authors have completed the ICMJE uniform disclosure form (available at https://atm. amegroups.com/article/view/10.21037/atm-22-220/coif). The authors have no conflicts of interest to declare.

Ethical Statement: The authors are accountable for all aspects of the work in ensuring that questions related to the accuracy or integrity of any part of the work are appropriately investigated and resolved. Experiments were performed under a project license (No. 20200637) granted by ethics board of The Fourth Military Medical University, in compliance with national guidelines for the care and use of animals.

Open Access Statement: This is an Open Access article distributed in accordance with the Creative Commons Attribution-NonCommercial-NoDerivs 4.0 International License (CC BY-NC-ND 4.0), which permits the noncommercial replication and distribution of the article with the strict proviso that no changes or edits are made and the original work is properly cited (including links to both the formal publication through the relevant DOI and the license). See: https://creativecommons.org/licenses/by-nc-nd/4.0/.

\section{References}

1. Sinna R, Boloorchi A, Mahajan AL, et al. What should define a "perforator flap"? Plast Reconstr Surg 2010;126:2258-63.

2. Koshima I, Soeda S. Inferior epigastric artery skin flaps without rectus abdominis muscle. Br J Plast Surg 1989;42:645-8.

3. Kim JT, Kim SW. Perforator Flap versus Conventional Flap. J Korean Med Sci 2015;30:514-22.

4. Zhang W, Li X, Li X. A systematic review and metaanalysis of perforator flaps in plantar defects: Risk analysis of complications. Int Wound J 2021;18:525-35.

5. O'Brien AL, Jadallah E, Chao AH. Reconstruction of a radical total vulvectomy defect with a single split anterolateral thigh perforator flap: A case report and review of the literature. Microsurgery 2021;41:70-4.

6. Khavanin N, Qiu C, Darrach H, et al. Intraoperative Perfusion Assessment in Mastectomy Skin Flaps: How Close are We to Preventing Complications? J Reconstr Microsurg 2019;35:471-8.

7. Nowak K, Karampinis I, Gerken ALH. Application of Fluorescent Dyes in Visceral Surgery: State of the Art and Future Perspectives. Visc Med 2020;36:80-7.

8. Schöpper S, Smeets R, Gosau M, et al. Intraoperative ICG-based fluorescence-angiography in head and neck reconstruction: Predictive value for impaired perfusion of free flaps. J Craniomaxillofac Surg 2021. [Epub ahead of print]. doi: 10.1016/j.jcms.2021.09.020.

9. Zhang $Z$, Wang $C$, Zhang $Z$, et al. Intraoperative Assessment of DIEP Flap Breast Reconstruction Using Indocyanine Green Angiography: Reduction of Fat Necrosis, Resection Volumes, and Postoperative Surveillance. Plast Reconstr Surg 2021;147:690e-1e.

10. Jakubietz RG, Schmidt K, Bernuth S, et al. Evaluation of the Intraoperative Blood Flow of Pedicled Perforator Flaps Using Indocyanine Green-fluorescence Angiography. Plast Reconstr Surg Glob Open 2019;7:e2462.

11. Karaaltin MV, Erdem A, Kuvat S, et al. Comparison of clinical outcomes between single- and multipleperforator-based free thoracodorsal artery perforator flaps: clinical experience in 87 patients. Plast Reconstr Surg 2011;128:158e-65e.

12. Reichenberger MA, Keil H, Mueller W, et al. Comparison of extracorporal shock wave pretreatment to classic surgical delay in a random pattern skin flap model. Plast Reconstr Surg 2011;127:1830-7.

13. Huemer GM, Froschauer SM, Pachinger T, et al. A comparison of pretreatment with a topical combination of nonivamide and nicoboxil and surgical delay in a random pattern skin flap model. J Plast Reconstr Aesthet Surg 2009;62:914-9. 
14. Abraham M, Badhey A, Hu S, et al. Thromboprophylaxis in Head and Neck Microvascular Reconstruction. Craniomaxillofac Trauma Reconstr 2018;11:85-95.

15. Bekara F, Herlin C, Somda S, et al. Free versus perforatorpedicled propeller flaps in lower extremity reconstruction: What is the safest coverage? A meta-analysis. Microsurgery 2018;38:109-19.

16. Kim JW, Kim DY, Ahn KM, et al. Surgical implications of anatomical variation in anterolateral thigh flaps for the reconstruction of oral and maxillofacial soft tissue defects: focus on perforators and pedicles. J Korean Assoc Oral Maxillofac Surg 2016;42:265-70.

17. Kwon JH, Lee KT, Lim SY, et al. Reliable transfer of multiple perforator-based pedicled flaps: Surgical technique and clinical outcomes. Microsurgery 2017;37:105-11.

18. Taylor GI, Palmer JH. The vascular territories (angiosomes) of the body: experimental study and clinical applications. Br J Plast Surg 1987;40:113-41.

19. Aydin MA, Mavili ME. Examining microcirculation improves the angiosome theory in explaining the delay phenomenon in a rabbit model. J Reconstr Microsurg 2003;19:187-94.

20. Pereira N, Valenzuela D, Mangelsdorff G, et al. Detection of Perforators for Free Flap Planning Using Smartphone Thermal Imaging: A Concordance Study with Computed Tomographic Angiography in 120 Perforators. Plast Reconstr Surg 2018;141:787-92.

21. Lohman RF, Ozturk CN, Ozturk C, et al. An Analysis of Current Techniques Used for Intraoperative Flap Evaluation. Ann Plast Surg 2015;75:679-85.

22. Rodrigues LM, Rocha C, Ferreira H, et al. Different lasers reveal different skin microcirculatory flowmotion - data from the wavelet transform analysis of human hindlimb perfusion. Sci Rep 2019;9:16951.

23. Sjøberg T, Mercer JB, Weum S, et al. The Value of Dynamic Infrared Thermography in Pedicled Thoracodorsal Artery Perforator Flap Surgery. Plast Reconstr Surg Glob Open 2020;8:e2799.

24. Parmeshwar N, Sultan SM, Kim EA, et al. A Systematic Review of the Utility of Indocyanine Angiography in Autologous Breast Reconstruction. Ann Plast Surg 2021;86:601-6.

25. Li K, Zhang Z, Nicoli F, et al. Application of Indocyanine Green in Flap Surgery: A Systematic Review. J Reconstr Microsurg 2018;34:77-86.

26. Li L, Gao N, Yang AQ, et al. Application of fluorescein combined with methylene blue in sentinel lymph node biopsy of breast cancer. Sci Rep 2021;11:12119.
27. Reinhart MB, Huntington CR, Blair LJ, et al. Indocyanine Green: Historical Context, Current Applications, and Future Considerations. Surg Innov 2016;23:166-75.

28. Matsui A, Lee BT, Winer JH, et al. Submental perforator flap design with a near-infrared fluorescence imaging system: the relationship among number of perforators, flap perfusion, and venous drainage. Plast Reconstr Surg 2009;124:1098-104.

29. Moyer HR, Losken A. Predicting mastectomy skin flap necrosis with indocyanine green angiography: the gray area defined. Plast Reconstr Surg 2012;129:1043-8.

30. Phillips BT, Lanier ST, Conkling N, et al. Intraoperative perfusion techniques can accurately predict mastectomy skin flap necrosis in breast reconstruction: results of a prospective trial. Plast Reconstr Surg 2012;129:778e-88e.

31. Jeon FHK, Varghese J, Griffin M, et al. Systematic review of methodologies used to assess mastectomy flap viability. BJS Open 2018;2:175-84.

32. Yang D, Morris SF. An extended dorsal island skin flap with multiple vascular territories in the rat: A new skin flap model. J Surg Res 1999;87:164-70.

33. Cederna PS, Chang P, Pittet-Cuenod BM, et al. The effect of the delay phenomenon on the vascularity of rabbit abdominal cutaneous island flaps. Plast Reconstr Surg 1997;99:183-93.

34. Maitz PK, Pribaz JJ, Duffy FJ, et al. The value of the delay phenomenon in flap prefabrication: an experimental study in rabbits. Br J Plast Surg 1994;47:149-54.

35. Dhar SC, Taylor GI. The delay phenomenon: the story unfolds. Plast Reconstr Surg 1999;104:2079-91.

36. Carroll WR, Esclamado RM. Ischemia/reperfusion injury in microvascular surgery. Head Neck 2000;22:700-13.

37. Fann DY, Lim YA, Cheng YL, et al. Evidence that NF$\kappa \mathrm{B}$ and MAPK Signaling Promotes NLRP Inflammasome Activation in Neurons Following Ischemic Stroke. Mol Neurobiol 2018;55:1082-96.

38. Chaudhry MA, Mercer JB, de Weerd L. In Vivo Perforasome Perfusion in Hemi-DIEP Flaps Evaluated with Indocyaninegreen Fluorescence Angiography and Infrared Thermography. Plast Reconstr Surg Glob Open 2021;9:e3560.

(English Language Editor: L. Huleatt)

Cite this article as: Wanyan $\mathrm{C}$, Wu Z, Zhang F, Li H, Yang Z, Wang J, Han X, Yang X, Lei D, Yang X, Wei J. An animal experiment study on the application of indocyanine green angiography in the harvest of multi-angiosome perforator flap. Ann Transl Med 2022;10(4):197. doi: 10.21037/atm-22-220 